\title{
Application of silk fibroin/chitosan/nano-hydroxyapatite composite scaffold in the repair of rabbit radial bone defect
}

\author{
PENG YE ${ }^{1}$, BIN YU ${ }^{2}$, JIANG DENG ${ }^{3}$, RONG-FENG SHE ${ }^{4}$ and WEN-LIANG HUANG ${ }^{5}$ \\ ${ }^{1}$ Department of Orthopaedics, Nanfang Hospital, First School of Clinical Medicine, Southern Medical University; \\ ${ }^{2}$ Department of Orthopaedic Trauma, The Southern Hospital Affiliated to Southern Medical University, Guangzhou, \\ Guangdong 510515; ${ }^{3}$ Department of Orthopaedics, The Third Hospital Affiliated to Zunyi Medical College, Zunyi, \\ Guizhou 563000; ${ }^{4}$ Department of Orthopaedics, Guizhou Provincial People's Hospital, Guiyang, Guizhou 550002; \\ ${ }^{5}$ Department of Orthopaedics, The Third Hospital Affiliated to Zunyi Medical College, Guizhou, Zunyi 563000, P.R. China
}

Received September 21, 2016; Accepted May 5, 2017

DOI: 10.3892/etm.2017.5231

\begin{abstract}
Silk fibroin (SF), chitosan (CS) and nano-hydroxyapatite (nHA) possess excellent biocompatibility, thus, these were used to construct a SF/CS/nHA composite scaffold. Previously published results identified that this material exhibited satisfactory physical and chemical properties, and therefore qualified as a repair material in bone tissue engineering. The aim of the present study was to investigate the capacity and mechanism of this composite scaffold in repairing bone defects. In total, 45 New Zealand white rabbits were used to model defect in the right radial bone. A radial bone defect was induced, and rabbits were divided into the following treatment groups ( $\mathrm{n}=15$ in each): Group A, in which the SF/CS/nHA scaffold was implanted; group $\mathrm{B}$, in which the SF/CS scaffold was implanted; and group C, in which rabbits did not receive subsequent treatment. X-ray scanning, specimen observation and histopathological examination were implemented at 1,2, 3 and 4 months after modeling, in order to evaluate the osteogenic capacity and mechanism. At 1 month after modeling, the bone density shadow in the X-ray scan was darker in group A as compared with that in group B. Observation of the pathological specimens indicated that normal bone tissues partially replaced the scaffold. At 2 months, the bone density shadow of group A was similar to normal bone tissues, and normal tissue began to replace the scaffold. At 3-4 months after modeling, the X-ray scan and histopathological observation indicated that the normal bone tissues completely replaced the scaffold in group A, with an unobstructed marrow cavity. However, the bone mass of group B was lower in comparison with that of group A. The bone defect induced in group $\mathrm{C}$ was filled with fibrous connective tissues. Therefore, it
\end{abstract}

Correspondence to: Dr Bin Yu, Department of Orthopaedic Trauma, The Southern Hospital Affiliated to Southern Medical University, 1838 North Guangzhou Road, Guangzhou, Guangdong 510515, P.R. China

E-mail: binyu_01@163.com

Key words: bone tissue engineering, composite scaffold, bone defect was concluded that the SF/CS/nHA composite scaffold may be a promising material for bone tissue engineering.

\section{Introduction}

The treatment of bone defects caused by trauma and tumors, in particular radial bone defect, is challenging in clinical practice. Approximately 800,000 bone repair surgeries with bone grafting are performed each year in the US alone. In China, $>30$ million bone defect cases are caused by trauma each year (1). However, the bone substitutes currently in use do not adequately combine the advantages of biocompatibility, high mechanical strength, easy degradation and low cost (2). With the developments in tissue engineering, tissue-engineered bones are being widely used as an alternative to allografts and autografts due to their low immunogenicity, absence of secondary trauma and easy availability $(3,4)$. Different scaffolds made of a single biomaterial have respective advantages and defects in vitro and in vivo. To address this problem, a compounding technique that adjusts the proportion of each material and the way of forming the composite is currently applied $(5,6)$. Budiraharjo et al (7) co-cultured a chitosan (CS) scaffold coated with nano-hydroxyapatite (nHA), and the stem cells and scaffold demonstrated excellent biocompatibility and osteoinductive capacity. Furthermore, Alves da Silva et al prepared a composite scaffold by polymerization of CS and silk fibroin (SF), which was then used for co-culture of bone marrow stem cells. This composite scaffold was capable of inducing chondrocyte differentiation (8).

An SF/CS composite scaffold was applied in the repair of knee joint defects in rabbits in a preliminary study (9). On the basis of good biocompatibility and appropriate degradation rates, the SF/CS/nHA composite scaffold with improved performance was prepared in the present study and used for the repair of defect in rabbit radial bone. The osteogenic capacity and mechanism were discussed in the present study using X-ray scanning and pathological observation.

\section{Materials and methods}

Materials. A total of 45 New Zealand white rabbits (age, 2 months; 24 females and 21 males; weight, $2.5 \pm 3.0 \mathrm{~kg}$ ) 
were obtained from the Zunyi Medical College Animal Experiment Center (Zunyi, China). Animals were housed at $15^{\circ} \mathrm{C}$ and $45 \%$ humidity, with ad libitum access to standard rabbit food and water. The experiments were conducted at the Central Laboratory of Zunyi Medical College. The study design involved randomized grouping of animals and in vivo experiments. The study was approved by the Ethics Committee of Zunyi Medical College.

Preparation of SF/CS/nHA composite scaffold. The $\mathrm{SF} / \mathrm{CS} / \mathrm{nHA}$ scafford was prepared as previously described (10). SF, CS and nHA were prepared into $2 \%$ solutions and then combined in three proportions, namely 1:1:0.5, 1:1:1 and 1:1:1.5. The mixture was stirred at $55^{\circ} \mathrm{C}$ using a magnetic stirrer until the bubbles disappeared and the mixing was uniform. Next, the mixture was transferred to a 9 -well plate using a $20 \mathrm{ml}$ syringe $(\sim 50 \mathrm{ml} /$ well $)$ and frozen at $-80^{\circ} \mathrm{C}$ for $24 \mathrm{~h}$. The frozen scaffolds transformed from fluid into solid state but still contained ice, so were sealed with parafilm. A pattern of holes was made on the parafilm so that the water could be absorbed. The scaffolds were placed in a vacuum drier for $36 \mathrm{~h}$. The scaffolds were then removed and soaked in a mixture of $75 \%$ methanol and $1 \mathrm{~mol} / \mathrm{l}$ sodium hydroxide for $15 \mathrm{~h}$. Subsequently, the scaffolds were repeatedly washed with ultra-pure water and dried again in the vacuum drier. The scaffolds were then placed for $10 \mathrm{~h}$ in a solution of crosslinking agents, containing 1-ethyl-3-(3-dimethylaminopropyl) carbodiimide and $\mathrm{N}$-hydroxysuccinimide. After rinsing, the wet composite scaffolds were frozen at $-80^{\circ} \mathrm{C}$ for $24 \mathrm{~h}$, followed by vacuum drying for 36 h. Finally, the prepared composite scaffolds were sealed for later use $(11,12)$.

Modeling. A total of 45 New Zealand white rabbits were fasted from food and water for $6 \mathrm{~h}$ before surgery. Anesthesia was induced by intravenous injection of $2.5 \%$ sodium pentobarbital via the ear margin. The rabbits were immobilized in the four limbs to prevent damage to the vessels or nerves caused by body twitching during surgery. Next, the right forelimb was exposed, the skin was disinfected conventionally with iodophor and alcohol, and incised from the midpoint of the radial bone. Dissection was performed layer by layer with caution in order not to damage the vessels or nerves. In the exposed radial bone, a defect of approximately $2 \mathrm{~cm}$ in length was performed using a sterilized saw blade, as previously described (13-15). After the radial bone defect was performed, rabbits were divided into three groups $(n=15$ each) and treated as follows: Group A, in which SF/CS/nHA composite scaffold was implanted; group B, in which SF/CS scaffold was implanted, serving as the negative control group; and group $\mathrm{C}$, in which no scaffold was implanted, serving as the blank control group (16). The incision was sutured layer by layer and disinfected with iodophor following surgery, and wrapped with gauze without applying external fixation. To prevent postoperative infection, penicillin $(500,000 \mathrm{U})$ and streptomycin $(0.1 \mathrm{~g})$ were injected intramuscularly. The rabbits were kept in separate cages subsequent to surgery.

Rough observation and X-ray scanning. The diet and mobility of rabbits were observed regularly subsequent to surgery, and the incisions were checked for reddening and infection. At 4, 6, 8 and 12 weeks after surgery, the rabbits were immobilized to obtain anteroposterior X-ray scans of the radial bone. Bony calluses and replacement of the scaffold by bone tissues were observed in X-ray scans. Rabbits were sacrificed and bone tissues were harvested at different times postoperatively (4, 6,8 and 12 weeks after surgery, $n=3$ at each time point) and made into pathological specimens to evaluate the repair of bone defects, the scaffold association with the surrounding tissues and the absorption of the scaffold.

Histopathological observation. Vertical and transverse sections were prepared for a comprehensive observation. Briefly, the specimens were fixed with $4 \%$ formaldehyde, decalcified and sliced into $4-\mu \mathrm{m}$ sections. Next, specimens were dewaxed in xylene, dehydrated through an ethanol gradient, washed with water and air dried for $24 \mathrm{~h}$. Pathological observation was based on hematoxylin-eosin (HE) and toluidine blue staining. The sections were dehydrated, sealed and observed under an inverted microscope to evaluate the growth of normal bone tissues, osteogenic capacity and degradation of the scaffold. The lesions in the surrounding tissues were also observed. Lane-Sandhu histologic criteria were used (17) i) Healing: None, 0; fiber, 1; fibrocartilage, 2; bone, 3; trabecular and cortical bone, 4. ii) Callus: None, 0; small amount of callus, 1; medium callus, 2; large amount of callus, 3; fully callus, 4. iii) Bone marrow: No bone marrow, 0 , bone marrow started, $1 ;>50 \%$ of the defect area has bone marrow, 2; complete marrow, 3; mature marrow, 4.

Statistical analysis. The results of rough observation, X-ray scan and pathological observation were analyzed using SPSS version 17.0 software (SPSS, Inc., Chicago, IL, USA). F-test was conducted to compare the mean values of multiple samples. Differences with $\mathrm{P}<0.05$ were considered to be statistically significant.

\section{Results}

Postoperative conditions and observations. Rabbits were provided with food $12 \mathrm{~h}$ after surgery in each group. The incisions healed within 1 week without severe infection, reddening or festering. At 1 week after surgery, the rabbits in groups A and B appeared to be more active compared with those in group $\mathrm{C}$, while the mobility was moderate in all groups 3 weeks later. As indicated by pathological observation, the scaffolds in groups A and B at 4 weeks after surgery were enclosed in fibrous connective tissues. The scaffolds under the periosteum presented signs of calcification and a grayish white color. Little difference was observed between groups A and B with the naked eye. At 8 weeks, the scaffold was gradually replaced by bony calluses in groups A and B. The scaffolds of group B had a light yellow color. Between weeks 12 and 16, osseointegration with a similar color and texture as that of the normal bone tissues was observed in group A. In group B, the scaffolds had a light yellow color, similar to that of the cartilage. Although the broken ends of the bone presented slight elongation at the early stage in group $\mathrm{C}$, no osseointegration was observed and the defect 
A

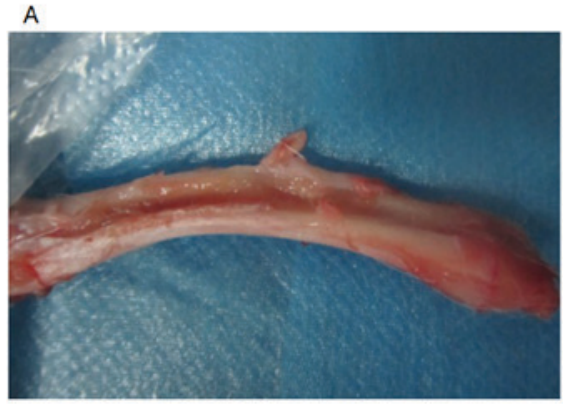

B

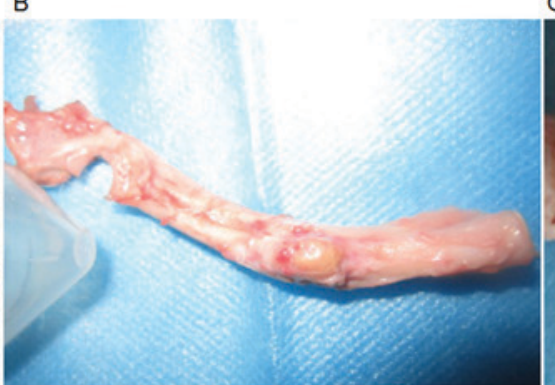

C

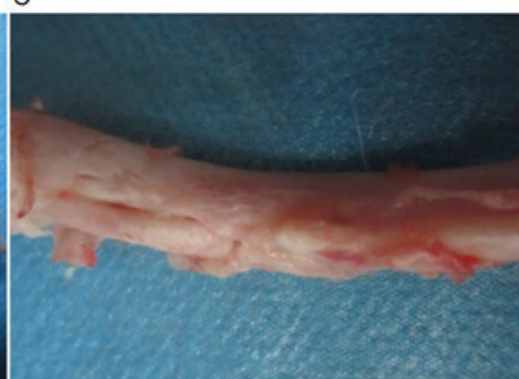

Figure 1. General observations of radial bone defect in rabbits treated with different scaffold materials after 16 weeks of implantation. Bone specimens are shown from rabbits with (A) SF/CS/nHA scaffold implantation (group A), (B) SF/CS implantation (group B), and (C) no treatment of the defect (group C). SF, silk fibroin; CS, chitosan; nHA, nano-hydroxyapatite.

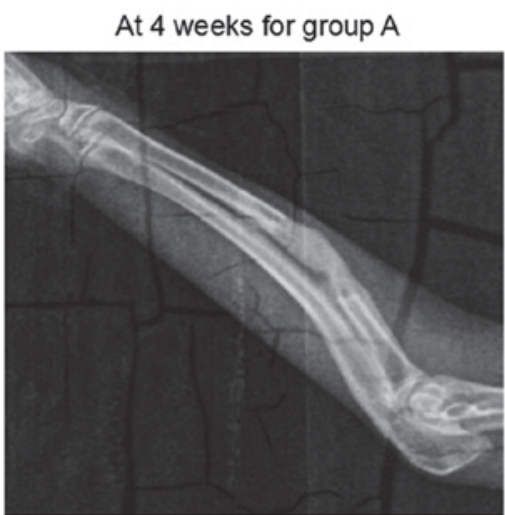

At 12 weeks for group $A$

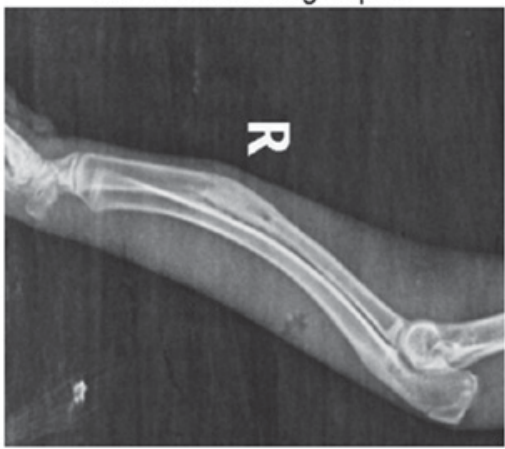

At 8 weeks for group $A$

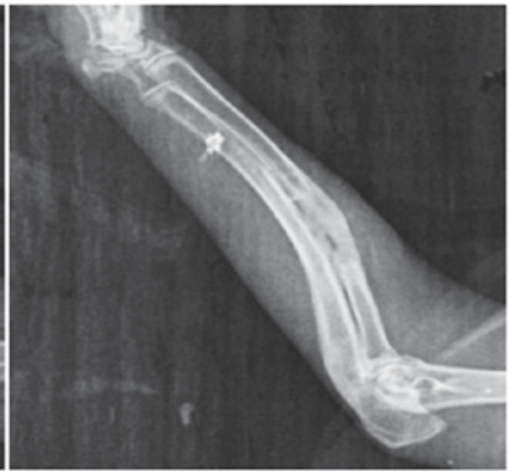

At 16 weeks for group $A$

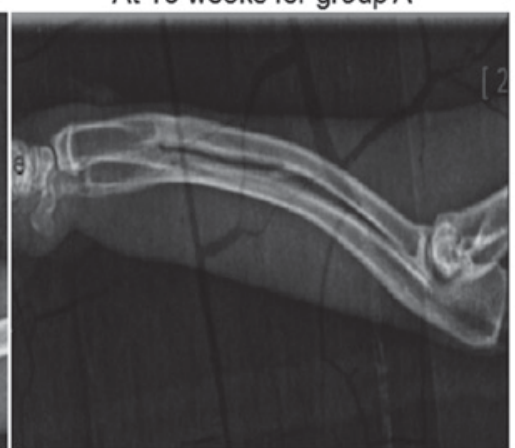

Figure 2. X-ray observation of rabbit radial bone defect in group A, after implantation of silk fibroin/chitosan/nano-hydroxyapatite composite scaffolds.

was filled with soft tissues during the entire observational period. Fig. 1 shows the radial bone defect of each group at 16 weeks after surgery.

Osteogenic capacity evaluated by X-ray scanning in each group. The results of X-ray examination conducted in group A are shown in Fig. 2. At 4 weeks after surgery, the scaffolds in the defect region presented with a grayish white high-density shadow. At 8 weeks after surgery, the calcified shadow was darkened with distinct boundary from the surrounding soft tissues. At 12 weeks, the marrow cavity was unobstructed partially between the two broken ends. Finally, at 16 weeks, no evident disparity was observed on the X-ray scans between the defect region and the normal bone tissue, while the marrow cavity was completely unobstructed.
As shown in Fig. 3, sporadic calcified shadows were observed in the scaffolds in group B at 4 weeks after surgery. These were clearly darker compared with the adjacent soft tissue shadows, though their morphology was less distinct. At 8 weeks, the calcified shadows were enhanced, although they remained lighter in color compared with normal bone tissues and were more distinct in morphology in comparison with the earlier X-ray scan. At 12 and 16 weeks after surgery, $\mathrm{X}$-ray scanning revealed slightly lighter bone density shadows compared with the normal bone tissues, and the marrow cavity was partially unobstructed.

As demonstrated in Fig. 4, new bone tissues grew from the two broken ends at 4 weeks in group $\mathrm{C}$, however, the length of growth was small. At 8 weeks, the calcified shadows at the two broken ends were enhanced, and the growth was 

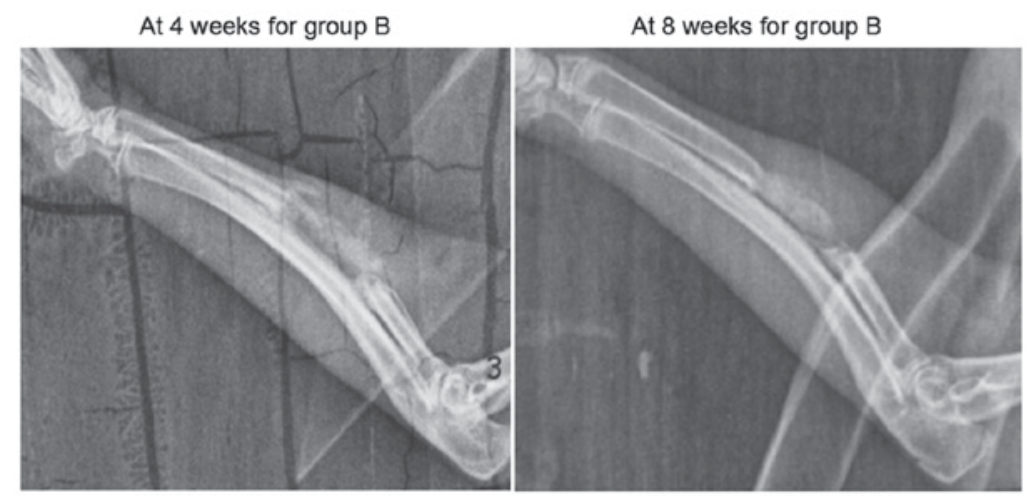

At 12 weeks for group B

At 16 weeks for group $B$

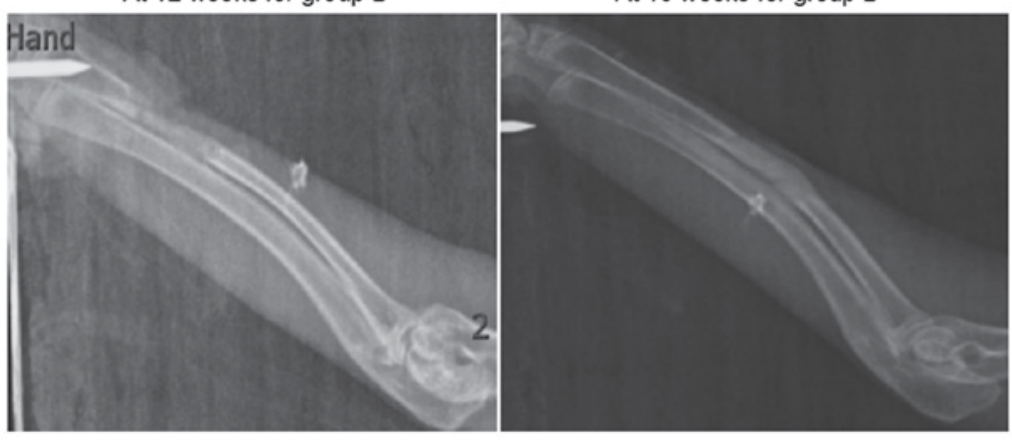

Figure 3. X-ray observation of rabbit radial bone defect in group B, after implantation of silk fibroin/chitosan composite scaffolds.

At 4 weeks for group $\mathrm{C}$

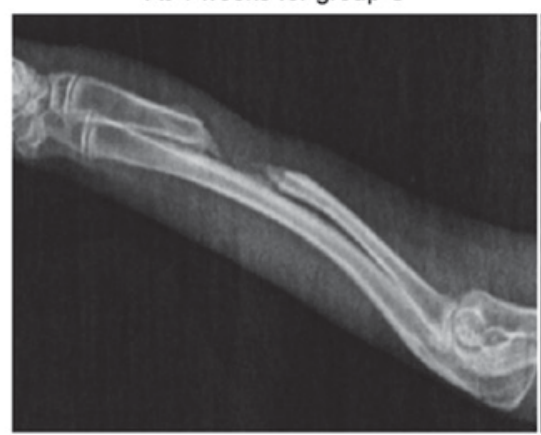

At 12 weeks for group C

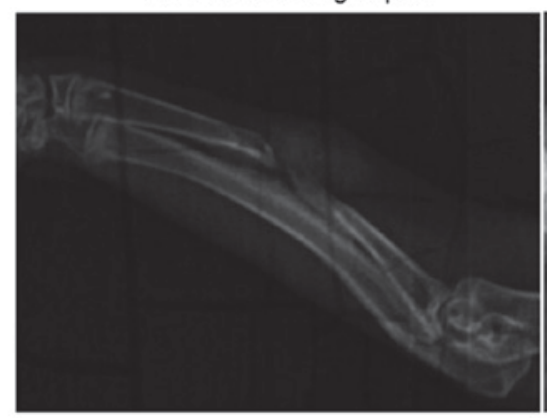

At 8 weeks for group $C$

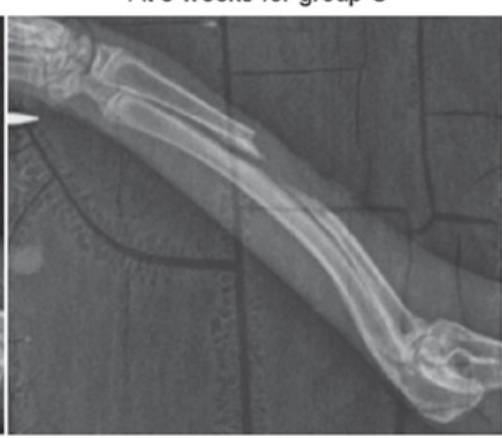

At 16 weeks for group C

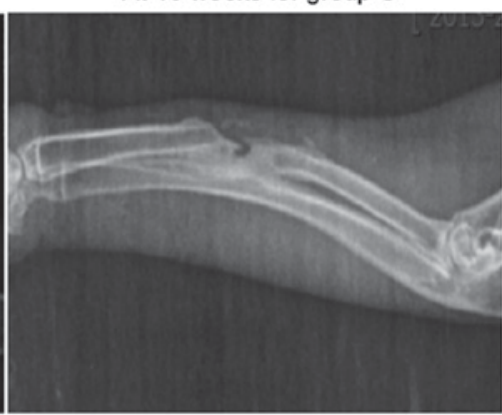

Figure 4. X-ray observations of radial bone defect in group $\mathrm{C}$ rabbits.

insignificant. In the blank region (defect), soft tissue shadows were observed. At 12 and 16 weeks after surgery, the elongation at the two broken ends appeared to have stopped, and the calcified shadows were enhanced to the extent of resembling the normal bone tissues. As the two broken ends were closed separately, bone nonunion occurred. Groups A and B both indicated osteogenesis in early stages, but in group A, higher
X-ray bone density was observed, indicating that group A had a better capacity for osteogenesis. Group C finally became bone nonunion.

Pathological observation. HE staining was performed in each group at 16 weeks. As seen from Fig. 5A, new bone tissues were observed in group A. Trabecular bones and 


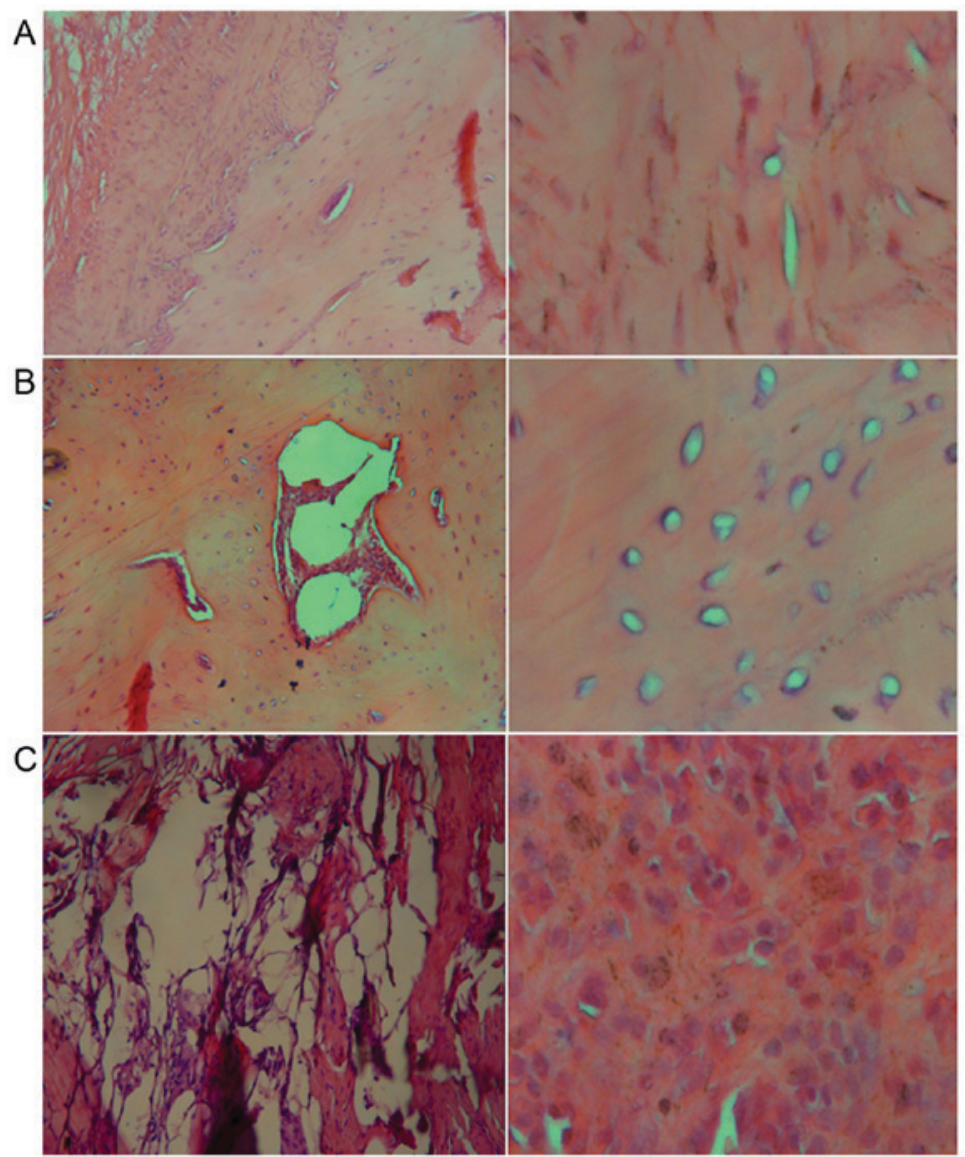

Figure 5. Hematoxylin-eosin staining of bone defect tissue after 16 weeks from surgery in rabbits with (A) SF/CS/nHA scaffold implantation (group A), (B) SF/CS implantation (group B), and (C) no treatment of the defect (group C). Magnification, x100. SF, silk fibroin; CS, chitosan; nHA, nano-hydroxyapatite.

long spindle-shaped osteocytes with deeply stained nuclei were observed under x100 magnification. The bone plate was distinct and the central canal, while bone matrix in lamellar arrangement was detected on the outside and the osteocytes. Under higher magnification, long spindle-shaped osteocytes were observed. In group B, the scaffolds were partially replaced by bone tissues and the osteocytes were surrounded by a considerable amount of chondrocytes (Fig. 5B). However, no trabecular bones or bone plates were observed, and large amounts of chondrocytes were observed under high magnification (Fig. 5B). Furthermore, in group C, large amounts of fibrous connective tissues were detected in contrast to a few bone-like tissues. Round and deeply-stained nuclei of myofibroblasts were observed under high magnification, which were larger in size compared with the osteocytes and vacuolated adipocytes (Fig. 5C). The Lane-Sandhu histological scores of each group are listed in Table I. Group A exhibited significantly higher Lane-Sandhu scores at each time point compared with groups B and C, indicating that the scaffold in group A achieved better osteogenesis.

\section{Discussion}

Bone tissue engineering involves three key aspects, including the presence of signaling molecules that induce bone tissue differentiation, appropriate scaffold materials and the ideal seed cells. Among these, the use of an appropriate scaffold material is the most important aspect, and this material is required to possess good mechanical strength combined with toughness. In normal bone tissues, the tough layer of connective tissues provides support. Thus, ensuring high mechanical strength in artificial scaffolds is essential. In order to repair different types of bone defects, scaffolds that are sufficiently strong and allow remodeling are required (18). Good biocompatibility with the host is essential, which refers to the absence of immune rejection risk, inflammation or even carcinogenesis following implantation. Three-dimensional (3D) scaffolds with reticular structure and large surface area of pores are preferred since these characteristics are conducive to the regeneration of bone tissues and vessels. A large surface area facilitates the growth and adhesion of the stem cells, and results in a more uniform degradation of the scaffolds. In addition, an appropriate degradation rate of the scaffolds is crucial (19). Long-term presence of scaffolds in the host will prevent regeneration and growth of bone tissues; however, if the scaffold degrades too fast, it cannot provide the support necessary for the repair of the bone defect $(20,21)$.

Among various materials used in tissue-engineered bones, natural polymers include collagen, CS and alginates, while artificial polymers include polylactic acid, polyphosphazenes and poly- $\beta$-hydroxybutyric acid. Furthermore, the main artificial inorganic materials used are hydroxyapatite, bioactive glass and $\beta$-tricalcium phosphate. Natural polymers are 
Table I. Lane-Sandhu scores in each group following scaffold implantation.

\begin{tabular}{lcccc}
\hline Group & 4 weeks & 8 weeks & 12 weeks & 16 weeks \\
\hline A & $6.50 \pm 0.56^{\mathrm{a}}$ & $8.75 \pm 0.48^{\mathrm{a}}$ & $9.85 \pm 0.14^{\mathrm{a}}$ & $10.54 \pm 0.13^{\mathrm{a}}$ \\
B & $3.95 \pm 0.38$ & $5.56 \pm 0.68$ & $7.25 \pm 0.51$ & $8.54 \pm 0.25$ \\
C & $2.54 \pm 0.31$ & $3.58 \pm 0.54$ & $4.39 \pm 0.42$ & $6.44 \pm 0.41$ \\
\hline
\end{tabular}

${ }^{\text {ap }}<0.05$, group A vs. other groups. Values are presented as the mean \pm standard deviation $(n=15)$. Rabbits underwent SF/CS/nHA scaffold implantation (group A), SF/CS scaffold implantation, or no treatment of the defect (group C). SF, silk fibroin; CS, chitosan; nHA, nano-hydroxyapatite.

superior in biocompatibility, but poor in mechanical strength. In addition, artificial polymers contain ingredients that mimic the inorganic components of normal bone, leading to the risk of inflammation and foreign body reaction. Artificial inorganic materials are superior in plasticity and mechanical strength, but poor in hydrophilicity and controllability of degradation rates (22). Therefore, in order to combine the advantages of different materials, composite scaffolds are constructed for bone tissue engineering (23-26).

In the study by Zhu et al (27), hydroxyapatite/calcium polyphosphate/poly-L-actic acid scaffolds were prepared from the raw materials of nHA, calcium polyphosphate and polylactic acid using a solvent casting, particle filtering method and gas forming method. The scaffolds had a 3D reticular structure with an average pore size of 200-400 $\mu \mathrm{m}$ and high modulus of elasticity. The mechanical performance was enhanced in the composite scaffold compared with the scaffold composed of a single material (27). Furthermore, Xiao et al (28) adopted a co-precipitation method and prepared composite bone cement using nHA, carboxymethyl CS and sodium alginate, which was then applied to the co-culture with rabbit bone marrow stromal cells in vitro. The cells adhered to the scaffold surface and proliferated continuously at days 2, 4, 6 and 8. Under a scanning electron microscope, pseudopods were observed in the cells that were adherent to the material surface, indicating good biocompatibility with the cells in vitro. In addition, Ye et al (29) prepared 3D scaffolds from PHBV by electrospinning technology, as well as bone substitute made of $\beta$-tricalcium phosphate alone. Application of the two scaffolds for the repair of rabbit tibial bone defect indicated that the composite scaffold had a superior repair effect after 8 weeks, while the degradation rate of the composite scaffold was more conducive to the repair (29). Furthermore, Yang et al (30) used SF and HA to prepare a composite scaffold for the repair of a rabbit articular cartilage defect. The articular cartilage was then harvested and observed to have uniformly and smoothly repaired the defect. CT examination indicated smooth articular surface and normal joint space, while observation of pathological specimens showed the filling of large amounts of chondrocytes and close binding to the cartilage (30). Mature trabecular bones and reticular structure were observed with complete repair of subchondral bone, thus indicating sufficient support and biocompatibility in vivo. Therefore, as compared with scaffolds composed of a single material, composite scaffolds can achieve the desired combination of properties by changing the proportion of each ingredient and the way of compounding. Although the SF/CS scaffold prepared in a preliminary study exhibited high biocompatibility in the repair of rabbit knee joint defect, the mechanical strength required further improvement (31-33). Therefore, another ingredient, namely nHA, was introduced in the scaffold used in the present study.

The SF/CS/nHA composite scaffold constructed in the current study had a 3D reticular structure with an average pore size of 100-200 $\mu \mathrm{m}$ and excellent physical and chemical properties. In order to test its performance, a defect measuring $1.5 \mathrm{~cm}$ in length was induced in the middle segment of rabbit radial bones. After incising the skin and exposing the radial bone, a large segmental bone defect was made with the removal of the periosteum. Surgery was performed using the same approach and procedures in all rabbits, and the length of the defect was four times larger than the diameter of the rabbit radial shaft. The severity of the defect was far beyond the scope of self-repair. The purpose of removing the periosteum was to prevent osteogenesis from the periosteum. Within 1 week after scaffold implantation, no reddening, fever or other signs of infection were observed in all groups. The incisions healed 2 weeks later, without festering or rejection. After 16 weeks, rabbits in group A did not suffer from any systemic reactions, such as anorexia, while vomiting and death were not reported. The aforementioned results of the present study indicated good biocompatibility of the scaffold. Furthermore, X-ray scans indicated enhanced calcified shadows over time in group A, with distinct morphology and margins. The defect region did not differ from the normal bone tissue on X-ray scans conducted 16 weeks after surgery, which suggested excellent osteoinductive and osteogenic effect of the scaffold. However, the calcified shadows in group B were evidently lighter compared with group A at the same time points, indicating poor osteogenic capacity. This may be due to the introduction of nHA in the scaffold used in group A, since degradation of nHA provides calcium and phosphorus ions that are required for osteogenesis $(34,35)$. In group $C$, the two broken ends of the bones were closed separately without osseointegration. Under low magnification, there were more osteoblasts in group A as compared with group B, and typical structures of bone tissues, such as trabecular bones and fibrous rings, were also observed. Under high magnification, group A had typical osteoblasts in the bone lacunae, while there were more round chondrocytes in group B. These observations provided convincing evidence that the addition of nHA enhanced the osteoinductive capacity.

In conclusion, the SF/CS/nHA composite scaffold demonstrated high biocompatibility and osteoinduction in the repair of a defect in rabbit radial bone. The degradation rate of the scaffolds was compatible with the regeneration of the bone tissues. Therefore, the SF/CS/nHA composite scaffold may qualify as a novel scaffold material, although the details of the repair mechanism currently remain unknown.

\section{Acknowledgements}

This study was supported by grants from the Guizhou Province Science and Technology Fund Project [no. SY (2010) 3101], the Guizhou Social Research Project [no. (2010) 015], and the Guizhou Province Governor Fund Project [no. (2011) (25)]. 


\section{References}

1. Xin Lei and SU Jia-can: Current status and prospect of artificial bone repair materials. J Traumatic Surg 3: 254-257, 2011.

2. Puppi D, Chiellini F, Piras AM and Chiellini E: Polymeric materials for bone and cartilage repair. Progress in Polymer Science 35: 403-440, 2010.

3. Pan Z and Ding JD: Poly (lactide-co-glycolide) porous scaffolds for tissue engineering and regenerative medicine. Interface focus 2: 366-377, 2012 .

4. Park SH, Park DS, Shin JW, Kang YG, Kim HK, Yoon TR and Shin JW: Scaffolds for bone tissue engineering fabricated from two different materials by the rapid prototyping technique: PCL versus PLGA. J Mater Sci Mater Med 23: 2671-2678, 2012.

5. WangZ,Li M,YuB,CaoL, Yang Q and Su J:Nanocalcium-deficient hydroxyapatite-poly (e-caprolactone)-polyethylene glycol-poly (e-caprolactone) composite scaffolds. Int J Nanomedicine 7: 3123-3131, 2012.

6. De Santis R, Gloria A, Russo T, D'Amora U, Zeppetelli S, Dionigi C, Sytcheva A, Herrmannsdörfer T, Dediu V and Ambrosio L: A basic approach toward the development of nanocomposite magnetic scaffolds for advanced bonetissue engineering. J Appl Polym Sci 122: 3599-3605, 2011.

7. Budiraharjo R, Neoh KG and Kang ET: Hydroxyapatite-coated carboxymethyl chitosan scaffolds for promoting osteoblast and stem cell differentiation. J Colloid Interface Sci 366: 224-232, 2012.

8. Alves da Silva ML, Crawford A, Mundy JM, Correlo VM, Sol P, Bhattacharya M, Hatton PV, Reis RL and Neves NM: Chitosan/polyester-based scaffolds for cartilage tissue engineering: Assessment of extracellular matrix formation. Acta Biomater 6: 1149-1157, 2010.

9. Deng J, Yu RF, Huang WL, Yuan C and Mo G: Restoration of cartilage defect with silk fibrin/chitosan biological scaffold compound by bone marrow mesenchymal stem cells in elderly rabbits. Chin J Geriatrics 31: 156-160, 2012.

10. Tiyaboonchai W, Chomchalao P, Pongcharoen S, Sutheerawattananonda $\mathrm{M}$ and Sobhon P: Preparation and characterization of blended Bombyx mori silk fibroin scaffolds. Fiber Polym 12: 324, 2011.

11. Murphy CM, Haugh MG and O'Brien FJ: The effect of mean pore size on cell attachment, proliferation andmigration in collagen-glycosaminoglycan scaffolds for bone tissue engineering. Biomaterials 31: 461-466, 2010.

12. Lu Q, Zhang X, Hu X and Kaplan DL: Green process to prepare silk fibroin/gelatin biomaterial scaffolds. Macromol Biosci 10 289-298, 2010

13. Zhang HF, Zhao CY, Fan HS, Zhang H, Pei FX and Wang GL: Histological and biomechanical study of repairing rabbit radius segmental bone defect with porous titanium. Beijing Da Xue Xue Bao 43: 724-729, 2011 (In Chinese).

14. Rahimzadeh R, Veshkini A, Sharifi D and Hesaraki S: Value of color Doppler ultrasonography and radiography for the assessment of the cancellous bone scaffold coated with nano-hydroxyapatite in repair of radial bone in rabbit. Acta Cir Bras 27: 148-154, 2012

15. Hao W, Dong J, Jiang M, Wu J, Cui F and Zhou D: Enhanced bone formation in large segmental radial defects by combining adipose-derived stem cells expressing bone morphogenetic protein 2 with nHA/RHLC/PLA scaffold. Int Orthop 34 $1341-1349,2010$

16. Deng J, She R, Huang W, Dong Z, Mo G and Liu B: A silk fibroin/chitosan scaffold in combination with bone marrow-derived mesenchymal stem cells to repair cartilage defects in the rabbit knee. J Mater Sci Mater Med 24: 2037-2046, 2013.

17. Huang Y, Huang D, Zhang D, Mou Y and Liu X: Promotion effect of FTY-720OP on treatment of bone defect with allograft bone by suppressing osteoclast formation and function. Zhongguo Xiu Fu Chong Jian Wai Ke Za Zhi 30: 426-431, 2016 (In Chinese).
18. Salerno M, Cenni E, Fotia C, Avnet S, Granchi D, Castelli F, Micieli D, Pignatello R, Capulli M, Rucci N, et al: Bone-targeted doxorubicin-loaded nanoparticles as a tool for the treatment of skeletal metastases. Curr Cancer Drug Targets 10: 649-659, 2010.

19. Yewle JN, Puleo DA and Bachas LG: Enhanced affinity bifunctional bisphosphonates for targeted delivery of therapeutic agents to bone. Bioconjug Chem 22: 2496-2506, 2011.

20. Kelly DJ and Jacobs CR: The role of mechanical signals in regulating chondrogenesis and osteogenesis of mesenchymal stem cells. Birth Defects Res C Embryo Today 90: 75-85, 2010.

21. Jayasuriya AC and Bhat A: Fabrication and characterization of novel hybrid organic/inorganic microparticles to apply in bone regeneration. J Biomed Mater Res A 93: 1280-1288, 2010.

22. Lee JS, Park WY, Cha JK, Jung UW, Kim CS, Lee YK and Choi SH: Periodontal tissue reaction to customized nano-hydroxyapatite block scaffold in one-wall intrabony defect: A histologic study in dogs. J Periodontal Implant Sci 42: 50-58, 2012.

23. Martínez-Vázquez FJ, Perera FH, Miranda P, Pajares A and Guiberteau F: Improving the compressive strength of bioceramic robocast scaffolds by polymer infiltration. Acta Biomater 6: 4361-4368, 2010.

24. Crouzier T, Sailhan F, Becquart P, Guillot R, Logeart-Avramoglou D and Picart C: The performance of BMP-2 loaded TCP/HAP porous ceramics with a polyelectrolyte multilayer film coating. Biomaterials 32: 7543-7554, 2011.

25. van der Pol U, Mathieu L, Zeiter S, Bourban PE, Zambelli PY, Pearce SG, Bouré LP and Pioletti DP: Augmentation of bone defect healing using a new biocomposite scaffold: An in vivo study in sheep. Acta Biomater 6: 3755-3762, 2010.

26. Liao F, Chen Y,LiZ, Wang Y, Shi B, Gong Z and Cheng X: A novel bioactive three-dimensional beta-tricalcium phosphate/chitosan scaffold for periodontal tissue engineering. J Mater Sci Mater Med 21: 489-496, 2010.

27. Zhu LY, Wang YP, Shi ZL and Zhang HM: Fabrication and properties of nanometer hydroxyapatite/calcium polyphosphate/poly (L-lactic acid) composite scaffold for bone tissue engineering. J Clin Rehabil Tissue Eng Res 16: 431-433, 2012.

28. Xiao HJ, Xue F, He ZM, Jin FQ and Shen YC: Preparation and characterization of nano-hydroxyapatite/carboxymethychitosan-sodium alginate bone cement composite material. J Clin Rehabil Tissue Eng Res 15: 7113-7117, 2011.

29. Ye R, Zhang XF, Yan HN, Pan YF, Huang NP, Lv LX and Jiang ZL: Hydroxybutyrate-hydroxyvalerate sodium meters of fiber material with bone defect repair. J Clin Rehabil Tissue Eng Res 16: 6284-6288, 2012.

30. Yang Y, Xu WY, Zhang Y, Zhang XX, Liu ZB, Qian H, Huang JP and Cui ZH: Symbol Silk fibroin/hydroxyapatite combined with bone marrow mesenchymal stem cells for construction of tissue engineered cartilage. J Clin Rehabil Tissue Eng Res 15: 5339-5342, 2011.

31. Rong Zijie, Yang Lianjun and Zhang Zanjie: The effect of nano-hydroxyapatite/collagen scaffolds incorporating ADM-PLGA microspheres in repairing the rabbits bone defects. J Pract Med 22: 3559-3562, 2014.

32. Ye Peng, Tian Ren-yuan and Ma Li-kun: Silk fibroin/chitosan/nano hydroxyapatite complicated scaffolds for bone tissue engineering. Chin J Tissue Eng Res 29: 5270-5274, 2013.

33. Ma Likun, Ye Peng and Jiang Deng: The cytotoxicity of silk fibroin/chitosan/nanohydroxyapatite bone tissue engineering scaffolds in vitro. Med J Westchina 8: 975-980, 2014.

34. Zhou H and Lee J: Nanoscale hydroxyapatite particles for bone tissue engineering. Acta Biomater 7: 2769-2781, 2011.

35. Zhang CY, Lu H, Zhuang Z, Wang XP and Fang QF: Nano-hydroxyapatite/poly (1-lactic acid) composite synthesized by a modified in situ precipitation: Preparation and properties. J Mater Sci Mater Med 21: 3077-3083, 2010. 